\title{
OPTIMAL CONSTRAINED CONTROL ALLOCATION IN MARINE SURFACE VESSELS WITH RUDDERS
}

\author{
Tor A. Johansen * Thomas P. Fuglseth * Petter Tøndel * \\ Thor I. Fossen * \\ * Department of Engineering Cybernetics, Norwegian University of \\ Science and Technology, NO-7491 Trondheim, Norway.
}

\begin{abstract}
A propeller with a rudder can produce a thrust vector within a range of directions and magnitudes in the horizontal plane for low-speed maneuvering and dynamic positioning. The set of attainable thrust vectors is non-convex because significant lift can be produced by the rudder only with forward thrust. We suggest to decompose the attainable thrust region into a finite union of convex polyhedral sets and derive a mixed-integer-like convex quadratic programming formulation of the optimal control allocation problem for marine vessels with any number of rudders as well as thrusters and other propulsion devices. Actuator rate and position constraints are explicitly taken into account. Using multi-parametric quadratic programming software, an explicit piecewise linear representation of the least-squares optimal control allocation law is pre-computed. It can be implemented with low computational complexity and high software reliability without the use of real-time optimization. The method is illustrated using a scale model ship in a basin.
\end{abstract}

Keywords: Ship control, multi-parametric programming, disjunctive programming.

\section{INTRODUCTION}

Control allocation algorithms for low-speed maneuvering and dynamic positioning of marine vessels have been developed for a variety of propulsion devices such as main propellers, tunnel thrusters, azimuth thrusters etc., (Fossen 2002, Sørdalen 1997, Berge and Fossen 1997, Webster and Sousa 1999, Lindfors 1993, Johansen et al. 2003). However, the use of rudders is not much studied, with the exception of the work of Lindegaard and Fossen (2003). Still, the use of rudders has many advantages, such as fuel efficiency, that makes them attractive to use for control allocation in joy-stick maneuvering as well as dynamic positioning.

Here we present a new control allocation method suited for marine surface vessels with one or more propellers with rudders. It is observed by Lindegaard and Fossen (2003) that due to asymmetric lift produced by the rudder with positive and negative thrust, the corresponding least-squares optimal control allocation problem becomes non-convex and therefore difficult

\footnotetext{
1 This work is in part supported by the Research Council of Norway by the Strategic University Program on Marine Cybernetics.

2 Corresponding author: tor.arne.johansen@itk.ntnu.no
}

to solve. The explicit solution approach suggested by Lindegaard and Fossen (2003) has the advantage that it provides a computationally simple and efficient solution. Here we extend this approach to the general case with any number of rudders and include actuator magnitude and rate constraints. Using convexification techniques, see (Balas 1998, Ceria and Soares 1999, Sherali and Adams 1999, Tawarmalani and Sahinidis 2002) for an overview, we suggest a convex mixed-integer-like reformulation of the problem that also takes into account actuator constraints, such as maximum force, rudder angle, and their rates of change. The optimization approach is based on solving several multi-parametric quadratic programs (mpQP) which allows explicit piecewise linear representations of the solution to be pre-computed (Bemporad et al. 2002, Tøndel et al. 2003a). A similar approach was suggested for computationally efficient constrained control allocation with non-rotatable propulsion devices by Johansen et al. (2002). The benefit of this is that the real-time implementation simply amounts to evaluating some pre-computed piecewise linear functions, which can be done efficiently and reliably using binary search tree representations of the piecewise linear functions (Tøndel et al. 2003b). The method is 
illustrated by examples using the experimental scale model ship CyberShip II.

\section{RUDDER CONTROL ALLOCATION MODELS}

The general formulation of the control allocation model for marine vessels in this section is taken from (Fossen 2002). In low-speed applications, it is common to use a 3-degrees-of-freedom model which only takes into account surge-, sway- and yaw-motions, with the corresponding vessel-fixed generalized forces $\tau=[X, Y, N]^{T}$. Propulsion devices (also called thrusters) include propellers (fixed or rotatable), azimuth thrusters, azipods and water-jets, among others. A fixed device is one which can only generate thrust along a single axis, while a rotatable device is one which can alter the direction of thrust. Propellers with rudders are included among the rotatable devices.

Assume a ship has a total of $p$ propulsion devices, divided among $p_{r}$ rotatable propulsion devices and $p_{f}$ fixed orientation propulsion devices. As a rotatable propulsion device has two controls (one for direction and one for magnitude of thrust), this gives us a total of $n=2 p_{r}+p_{f}$ control variables. The propulsion device with index $k$ is located at a position $r_{k}=\left[\ell_{k, x}, \ell_{k, y}\right]^{T}$ relative to the center of rotation in the vessel-fixed coordinate system. The device produces a force $T_{k}$ in a direction defined by the angle $\alpha_{k}$. For fixed orientation devices, $\alpha_{k}$ is constant, while for rotatable devices it can be altered. The propulsion device with index $k$ provides the following contribution to the generalized forces acting on the ship:

$$
\begin{aligned}
X_{k} & =T_{k} \cdot \cos \alpha_{k} \\
Y_{k} & =T_{k} \cdot \sin \alpha_{k} \\
N_{k} & =T_{k} \cdot m_{k}
\end{aligned}
$$

with $m_{k}=\ell_{k, x} \cdot \sin \alpha_{k}-\ell_{k, y} \cdot \cos \alpha_{k}$. The sum of forces is then given by:

$$
\tau=A(\alpha) \cdot T
$$

where $T=\left[T_{1}, \ldots, T_{p}\right]^{T}$ and

$$
A(\alpha)=\left[\begin{array}{ccc}
\cos \alpha_{1} & \cdots & \cos \alpha_{p} \\
\sin \alpha_{1} & \cdots & \sin \alpha_{p} \\
m_{1} & \cdots & m_{p}
\end{array}\right]
$$

It is useful to apply the concept of extended thrust. The extended thrust vector $u$ is found by decomposing the individual thrust vectors in the horizontal plane according to: $u_{k, x}=X_{k}, u_{k, y}=Y_{k}$. For a rotatable propulsion device, $u_{k}=\left[u_{k, x}, u_{k, y}\right]^{T} \in \mathbb{R}^{2}, 1 \leq k \leq$ $p_{r}$, while for a fixed propulsion device $u_{k} \in \mathbb{R}$ and $p_{r}+1 \leq k \leq p$. When using the concept of extended thrust, (Sørdalen 1997), the generalized thrust vector is given by a linear model

$$
\tau=B u
$$

where $u=\left[u_{1}, u_{2}, \ldots, u_{p}\right]^{T} \in \mathbb{R}^{n}$ and $B \in \mathbb{R}^{3 \times n}$ is given by:

$$
B=\left[B_{r}, B_{f}\right]
$$

$B_{r} \in \mathbb{R}^{3 \times 2 p_{r}}$ is defined as:

$$
B_{r}=\left[\begin{array}{ccccc}
1 & 0 & \cdots & 1 & 0 \\
0 & 1 & \cdots & 0 & 1 \\
-\ell_{1, y} & \ell_{1, x} & \cdots & -\ell_{p_{r}, y} & \ell_{p_{r}, x}
\end{array}\right]
$$

and $B_{f} \in \mathbb{R}^{3 \times p_{f}}$ is:

$$
B_{f}=\left[\begin{array}{ccc}
\cos \alpha_{p_{r}+1} & \cdots & \cos \alpha_{p} \\
\sin \alpha_{p_{r}+1} & \cdots & \sin \alpha_{p} \\
m_{p_{r}+1} & \cdots & m_{p}
\end{array}\right]
$$

In the rest of this paper we focus mainly on propellers with rudders, although the method can be used with any combination of fixed and rotatable propulsion devices.

We now briefly describe the propeller/rudder model given in Lindegaard and Fossen (2003):

$$
\begin{aligned}
& \mathbb{T}= \begin{cases}k_{T p} \omega^{2}, \quad \omega \geq 0 \\
k_{T n}|\omega| \omega, \omega<0\end{cases} \\
& L= \begin{cases}\mathbb{T}\left(1+k_{L n} \omega\right)\left(k_{L \delta_{1}} \delta+k_{L \delta_{2}}|\delta| \delta\right), & \omega \geq 0 \\
0, & \omega<0\end{cases} \\
& D= \begin{cases}\mathbb{T}\left(1+k_{D n} \omega\right)\left(k_{D \delta_{1}}|\delta|+k_{D \delta_{2}} \delta^{2}\right), & \omega \geq 0 \\
0, & \omega<0\end{cases}
\end{aligned}
$$

$\mathbb{T}$ is the nominal thrust, $L$ is the rudder lift force, $D$ is the rudder drag, $\omega$ is the propeller angular velocity, and $\delta$ is the rudder angle. The surge and sway forces of each thruster are given as follows:

$$
X=\mathbb{T}-D, \quad Y=L
$$

This model gives rise to a fan-shaped attainable thrust region when the thrust force is positive, see Figure 1 for an example corresponding to the model ship CyberShip II, (Lindegaard and Fossen 2003). When the thrust is negative, the rudders have no significant effect, and the thrust region is simply a line along the $X$-axis. Consequently, the attainable thrust region is inherently non-convex since no sway force can be produced with negative surge force.

\section{CONVEX OPTIMIZATION FORMULATION}

The objective of the control allocation scheme is to translate a commanded generalized force vector $\tau_{c}$ into a set of control signals. In this paper, the objective is to find a control allocation scheme that yields an extended thrust vector $u$ inside the attainable thrust region that is optimal with respect to some quadratic cost function in $u$ and admissible with respect to all actuator constraints. This leads to the following criterion, where the first term is a measure of the use of control energy

$$
J(u, s)=u^{T} H u+\left(u-u_{0}\right)^{T} M\left(u-u_{0}\right)+s^{T} Q s
$$




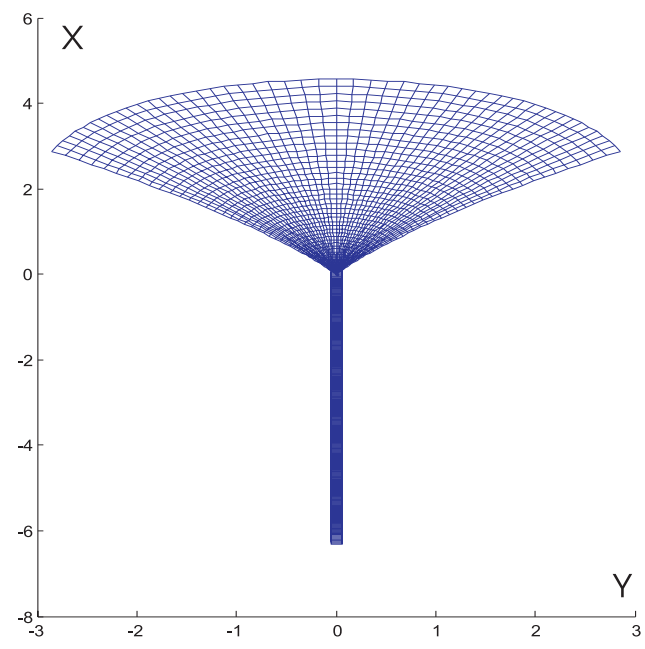

Fig. 1. Attainable thrust region for propeller with rudder. $X$ is the surge force and $Y$ is the sway force.

with non-negative matrices $H, Q$ and $M$, subject to the following constraints

$$
\begin{array}{r}
\tau_{c}=B u+s \\
-\epsilon X_{k} \leq Y_{k}-Y_{k, 0} \leq \epsilon X_{k}, \text { if } X_{k}>0 \\
u \in \bigcup_{i}\left\{u \in \mathbb{R}^{p} \mid A_{i} u \leq b_{i}\right\}
\end{array}
$$

where $\epsilon>0$ and the extended thrust at the previous sample is $u_{0}$. The objective and constraints are described in the rest of this section.

In order to get a solution also when meeting the commanded thrust requirement is impossible, we introduce slack variables $s=\left[s_{x}, s_{y}, s_{\psi}\right]^{T}$. They account for the difference between commanded forces $\tau_{c}$ and actual forces $\tau$, cf. (11). Typically, we want $s$ to be zero if a feasible solution exists. Therefore, the weighting of $s$ will need to be much higher than the weighting of $u$. Decreasing the weighting of $s$ leads to a solution that does not accurately give the desired thrust.

The cost function (10) penalizes the changes in extended thrust, rather than in thruster control signals. The relationship between thrust and thruster control signals such as rudder angles and propeller speed/pitch is non-linear, as described in Section 2, so even if thruster forces change in a smooth fashion, the control signals may experience large variations and even discontinuities. Particularly, when the surge force is close to zero, small changes in sway force may still lead to abrupt changes in rudder angle. If we wish to limit rudder rate, it is not sufficient to simply limit the rate of change in sway force. Instead, we attempt to limit the change in thrust vector angle. For each of the thrusters, we wish to add the following constraint:

$$
\left|\frac{Y_{k}}{X_{k}}-\frac{Y_{k, 0}}{X_{k, 0}}\right| \leq \epsilon
$$

With the assumption $X_{k} \approx X_{k, 0}$ the constraint (12) is a linear approximation to (14).
As discussed in Lindegaard and Fossen (2003) the non-convex nature of the feasible thrust region makes it in general difficult to solve the above optimization problem. However, this difficulty can be avoided by approximating the non-convex region in Figure 1 as union of two convex regions, corresponding to positive and negative values of $X$, respectively. This is a well known convexification technique often referred to as disjunctive programming (Balas 1998, Ceria and Soares 1999, Sherali and Adams 1999, Tawarmalani and Sahinidis 2002). For computational convenience, each of the convex regions are approximated with polyhedra with a sufficient degree of accuracy, see (13). For example, a vessel with two propellers and rudders leads to a union of $2^{2}=4$ convex polyhedral sub-regions, corresponding to all combinations of positive and negative surge force values:

(1) $X_{1} \geq 0, X_{2} \geq 0$.

(2) $X_{1}<0, X_{2} \geq 0$.

(3) $X_{1} \geq 0, X_{2}<0$.

(4) $X_{1}<0, X_{2}<0$.

An optimal constrained control allocation problem can therefore be decomposed into a finite number of convex quadratic programs, corresponding to minimizing (10) subject to (11) and (12) within polyhedral attainable thrust sub-region represented by some linear inequalities $A_{i} u \leq b_{i}$ corresponding to a single subregion of the union (13). At the final step the optimal solution must be chosen among all the solutions of these convex problems. This is referred to as the integer part of the solution, because of its resemblance to mixed-integer optimization. The case with two propellers with rudders is illustrated in Figure 2. For given extended thrusts $X_{k}$ and $Y_{k}$ the propeller/rudder model in section 2 must be inverted in the final block in Figure 2.

The quadratic programming sub-problems described above are in fact multi-parametric quadratic programming (mp-QP) problems, as they can be formulated as

$$
\begin{aligned}
& \min _{u, s} J(u, s), \\
& \text { subject to } \tau_{c}=B u+s, \mathbb{A}_{i} u \leq \mathbb{B}_{i}+\mathbb{C}_{i} u_{0}
\end{aligned}
$$

for some appropriate matrices $\mathbb{A}_{i}, \mathbb{B}_{i}$ and $\mathbb{C}_{i}$. This is an optimization problem with free variables $u$ and $s$, where $u_{0}$ and $\tau_{c}$ enter as parameters. Because the cost is quadratic and the constraints are linear (in both the free variables and the parameters), the solution to such mp-QP problems can be pre-computed and represented in an explicit form as functions of $\tau_{c}$ and $u_{0}$. More specifically, the optimal solutions $u$ and $s$ are given as a piecewise linear function of the parameters $\tau_{c}$ and $u_{0}$. We use the mp-QP algorithm described by Tøndel et al. (2003a).

As observed in Lindegaard and Fossen (2003) the least-squares control allocation problem in the case of propellers with rudders does not always have a unique solution. The reason for this is essentially the non-convex nature of the thrust region. Such lack of uniqueness gives rise to a discontinuity of the solution. 


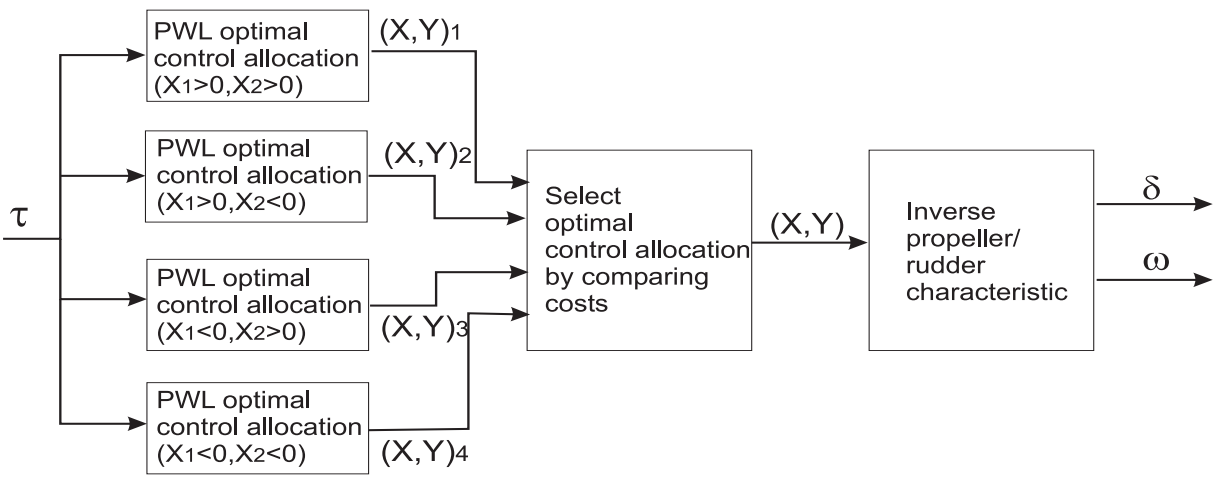

Fig. 2. Constrained control allocation approach.

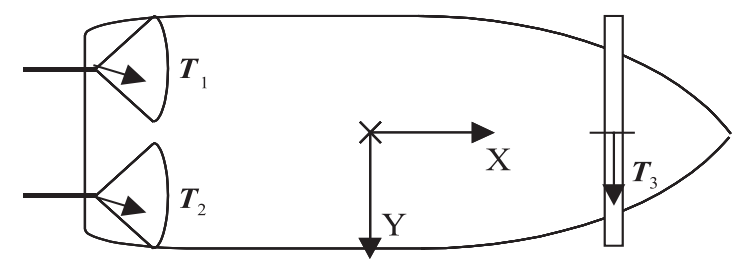

Fig. 3. Thruster configuration, CyberShip II.

A small change in the commanded generalized force $\tau_{c}$ may lead to undesirable chattering of the rudders between two or more solutions, corresponding to different configurations of positive/negative propellers speeds (or surge forces), that have the same cost. In order to reduce the amount of chattering we introduce a hysteresis such that the integer part of the solution is allowed to switch only if this leads to significant reduction in the cost function compared to the integer solution at the previous sample.

The objective of the rate constraint (12) is to avoid large changes in control signals. However, if the surge thrust $X_{k}$ is close to zero, even a very small change in the sway thrust $Y_{k}$ may require a large change in the rudder angle. Similar to Lindegaard and Fossen (2003) we therefore implement a regularization by shifting the fan-shaped thrust region in Figure 1 slightly into the area with positive $X_{k}$. As a consequence, the control allocation algorithm will require smoother changes in the rudder angle at the cost of some error near zero thrust, which is usually acceptable.

\section{TEST RESULTS}

The experiments described in this paper were performed with a model supply vessel, called CyberShip II. This is an approximately 1:70 scale replica of an offshore supply vessel, with an overall length of $1.22 \mathrm{~m}$. The vessel is equipped with three propulsion devices: two stern propellers, each with an accompanying rudder, and one bow-mounted tunnel thruster. All propulsion devices are rpm-controlled. The bow thruster can only provide thrust in the sway-direction, while the aft propellers/rudders can provide thrust in both surge- and sway-directions. The thruster configuration is illustrated in Figure 3, and model parameters and further details are given in Lindegaard and Fossen (2003). The experiments are carried out in the Marine Cybernetics Laboratory at NTNU, Trondheim. The sampling interval of the control allocation is $0.1 \mathrm{~s}$.

The extended force vector for CyberShip II is $u=$ $\left[X_{1}, Y_{1}, X_{2}, Y_{2}, Y_{3}\right]^{T}$. The tuning of the optimization problem is

$$
\begin{aligned}
M & =\operatorname{diag}(10,0,10,0,10) \\
H & =\operatorname{diag}(1,1,1,1,5) \\
Q & =\operatorname{diag}\left(10^{3}, 10^{3}, 10^{4}\right)
\end{aligned}
$$

and $\epsilon=0.1$. The rudder angles as constrained to \pm 35 deg, and the propeller speeds to $\pm 35 \mathrm{~Hz}$.

The solution consists of four different search trees, resulting from the four piecewise linear mp-QP solutions. In order to give an indication of the computational complexity of the approach, the most complex of the four search trees is the one representing the solution where both aft propellers give forward thrust. This search tree has 23395 nodes, 200 constituent linear functions, and 54486 stored numbers. At most 407 arithmetic operations are needed to compute the corresponding control allocation.

Figure 4 shows a sequence of commanded generalized forces, and Figure 5 shows the control signals, computed by the suggested control allocation method. The commanded and achieved generalized forces are almost indistinguishable, and the commanded control signals are continuous, with no undesirable chattering. The integer solutions are as follows: $\left(X_{\text {port }}<0, X_{\text {starb }}<0\right)$ in samples 0 to $5,\left(X_{\text {port }}<0, X_{\text {starb }}>0\right)$ in samples 6 to 100 , $\left(X_{\text {port }}<0, X_{\text {starb }}<0\right)$ in samples 101 to 119 , and $\left(X_{\text {port }}>0, X_{\text {starb }}<0\right)$ in samples 120 to 300 . The hysteresis is set to $10 \%$, such that the integer variables are allowed to switch only if $10 \%$ or more improvement in cost can be achieved.

Experimental results are shown in Figures 6 - 8. The test consists of commanding a small sway force with the joystick. Figure 7 shows the resultant movement. The yaw angle stays nearly constant. The position 

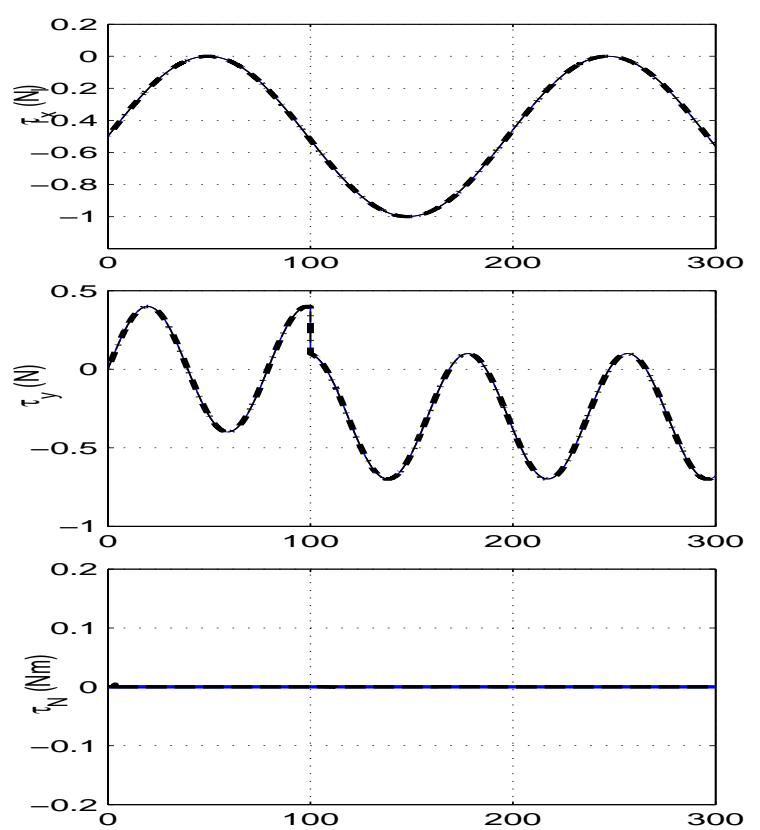

Fig. 4. Simulation results: Commanded generalized forces (solid), and achieved generalized forces with the suggested method (dashed).

and yaw angle is given in an Earth-fixed coordinate frame. It was necessary to apply some yaw moment to maintain heading, since the thruster model may not be entirely accurate, and in particular the influence of hydrodynamic forces are not accounted for in the model. Figure 8 shows the control signals given by the control allocation system. It is easily seen that these are close to constant, and that the curves are fairly smooth, with no sign of chattering. As long as the commanded force follows a more or less smooth curve, the actuator commands will do so too.

\section{CONCLUSIONS}

An optimal constrained control allocation strategy for marine vessels with rudders is suggested. The nonconvexity of the problem is tackled by a mixedinteger-like convex re-formulation that allows the solution to be pre-computed in a piecewise linear form using multi-parametric quadratic programming. A weakness of the approach is that the pre-computed explicit solution does not easily admit reconfiguration, unless several cases are pre-computed. Still, the computational efficiency and performance of the approach, which have been demonstrated using scale model ship experiments, indicate that the approach can be used efficiently in practise. Additional experimental results, including trajectory maneuvering and dynamic positioning, are given in Fuglseth (2003). It is also demonstrated in Fuglseth (2003) that the performance is comparable to Lindegaard and Fossen (2003).
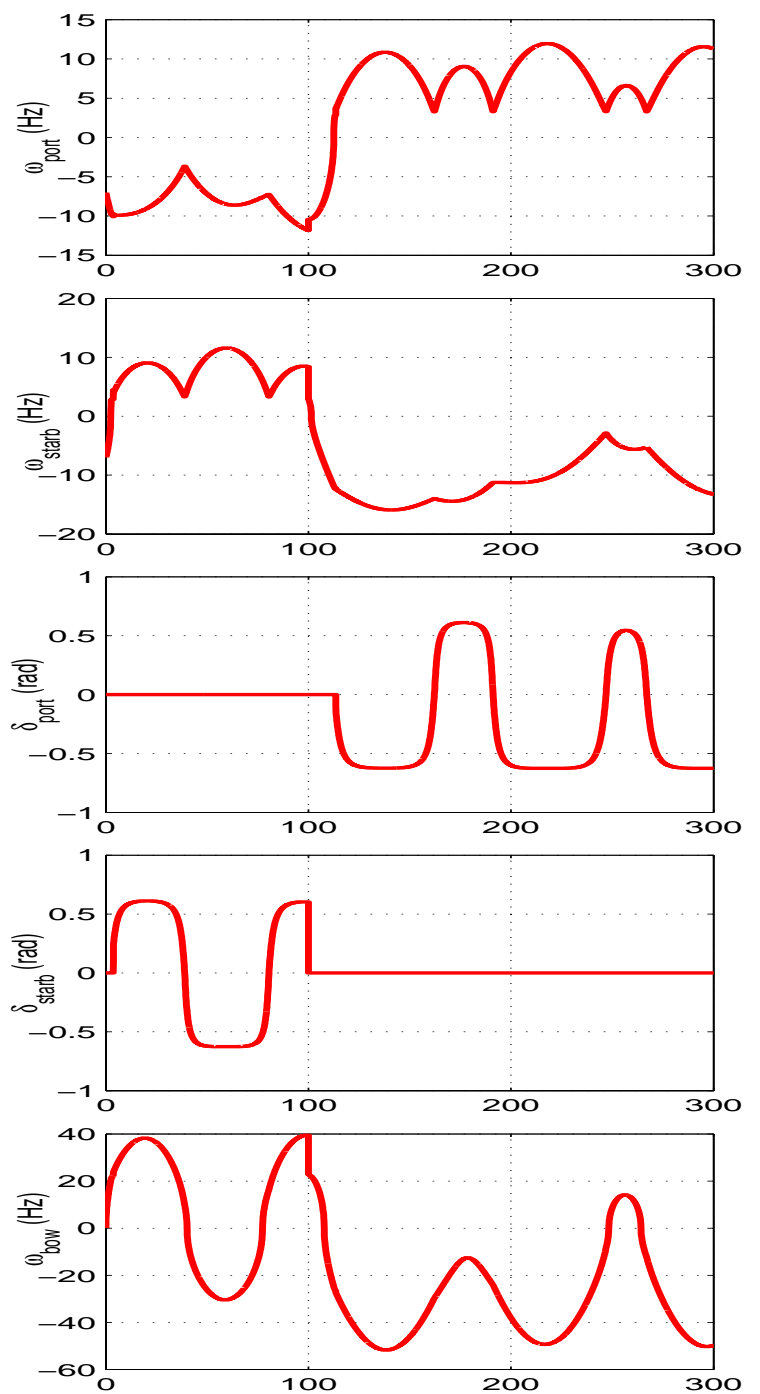

Fig. 5. Simulation results: Control signals.
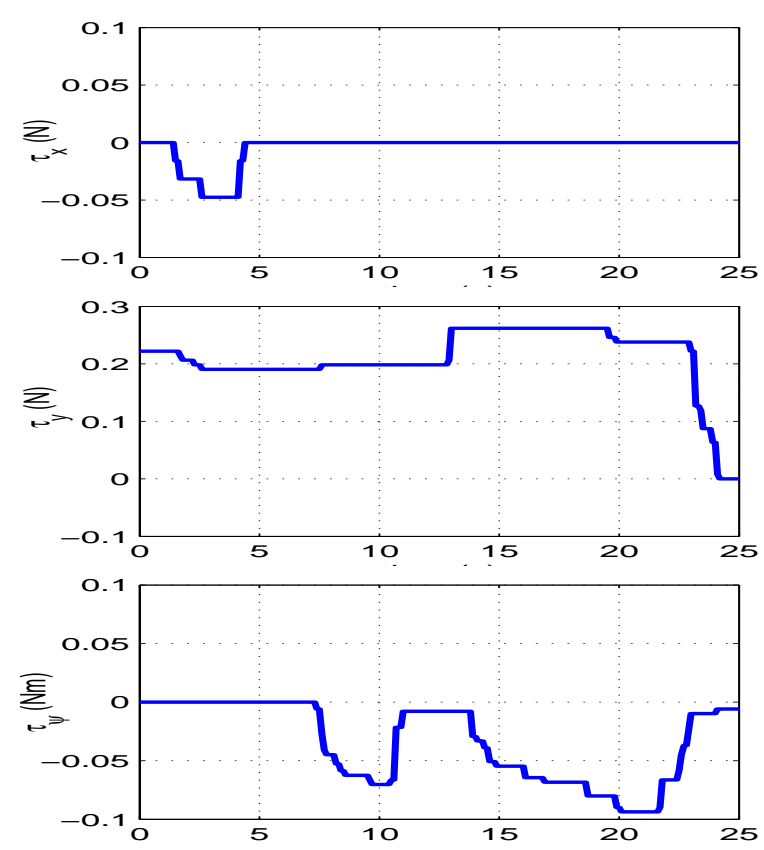

Fig. 6. Experimental results: Commanded generalized forces with joystick. 


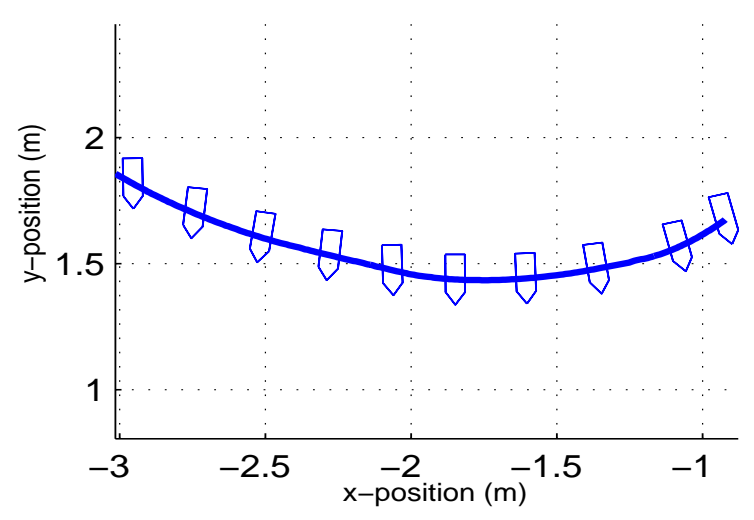

Fig. 7. Experimental results: $(x, y)$-position and yawangle.
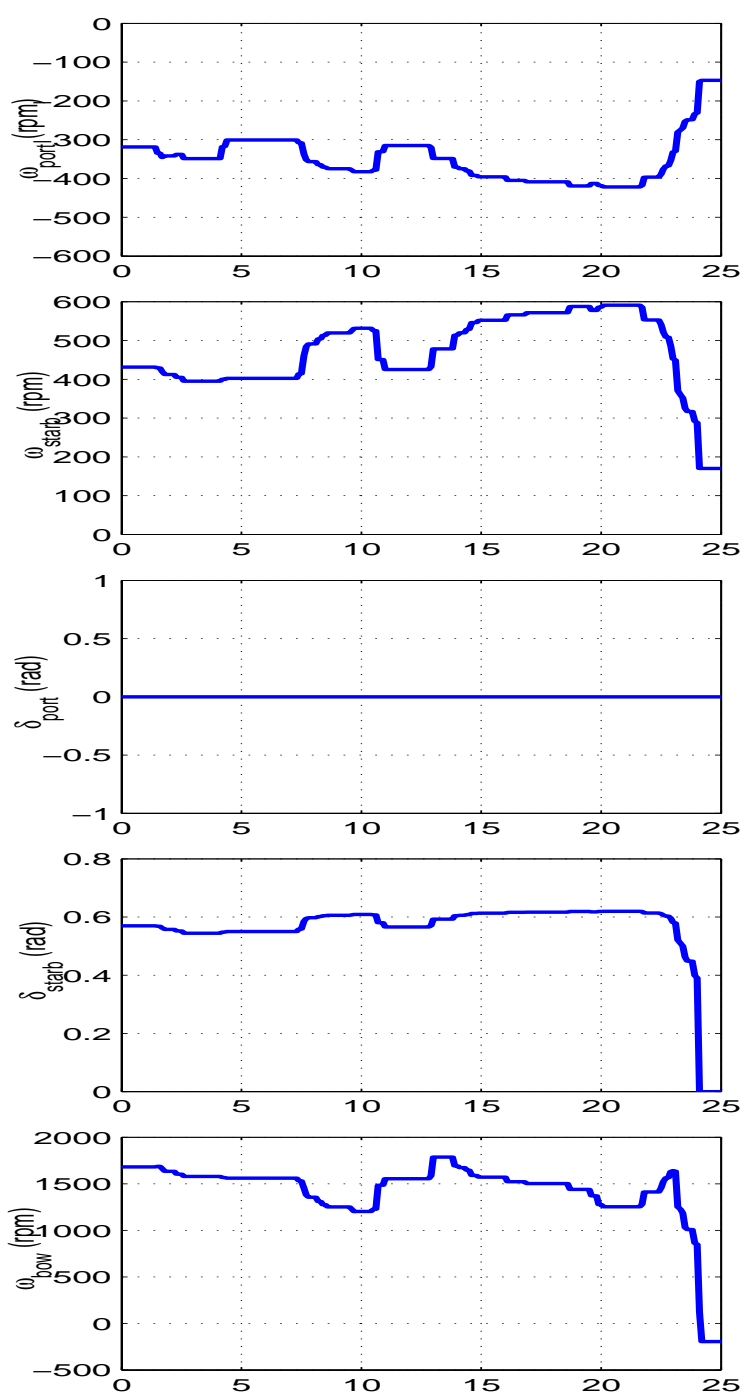

Fig. 8. Experimental results: Control signals.

\section{REFERENCES}

Balas, E. (1998). Disjunctive programming: Properties of the convex hull of feasible points. Discrete Applied Mathematics 89, 3-44.

Bemporad, A., M. Morari, V. Dua and E. N. Pistikopoulos (2002). The explicit linear quadratic regulator for constrained systems. Automatica 38, 3-20.
Berge, S. P. and T. I. Fossen (1997). Robust control allocation of overactuated ships; experiments with a model ship. In: Preprints IFAC Conference on Maneuvering and Control of Marine Craft, Brijuni, Croatia.

Ceria, S. and J. Soares (1999). Convex programming for disjunctive convex optimization. Math. Programming 86, 595-614.

Fossen, T. I. (2002). Marine Control Systems: Gudiance, Navigation and Control of Ships, Rigs and Underwater Vehicles. ISBN 82-92356-00-2, Marine Cybernetics, Trondheim.

Fuglseth, T. P. (2003). Optimal thrust allocation using rudders. M.Sc. thesis, Department of Engineering Cybernetics, Norwegian University of Science and Technology, Trondheim, Norway.

Johansen, T. A., T. I. Fossen and P. Tøndel (2002). Optimal constrained control allocation via multiparametric programming. J. Guidance, Control and Dynamics, submitted for publication.

Johansen, T. A., T. I. Fossen and S. P. Berge (2003). Constrained nonlinear control allocation with singularity avoidance using sequential quadratic programming. IEEE Trans. Control Systems Technology, accepted for publication.

Lindegaard, K.-P. and T. I. Fossen (2003). Fuel efficient control allocation for surface vessels with active rudder usage: Experiments with a model ship. IEEE Trans. Control Systems Technology, accepted.

Lindfors, I. (1993). Thrust allocation methods for the dynamic positioning system. In: Proc. 10th International Ship Control Symposium, Ottawa, Canada. pp. 3.93-3.106.

Sherali, H. D. and W. P. Adams (1999). A reformulation-linearization technique for solving discrete and continuous nonconvex problems. Nonconvex optimization and its applications, Volume 31, Kluwer Academic Press, Dordrecht.

Sørdalen, O. J. (1997). Optimal thrust allocation for marine vessels. Control Engineering Practice 5, 1223-1231.

Tawarmalani, M. and N. V. Sahinidis (2002). Convexification and global optimization in continuous and mixed-integer nonlinear programming: Theory, algorithms, software, and applications. Nonconvex optimization and its application, Volume 65, Kluwer Academic Press, Dordrecht.

Tøndel, P., T. A. Johansen and A. Bemporad (2003a). An algorithm for multi-parametric quadratic programming and explicit MPC solutions. Automatica 39, 489-497.

Tøndel, P., T. A. Johansen and A. Bemporad (2003b). Evaluation of piecewise affine control via binary search tree. Automatica 39, 945-950.

Webster, W. C. and J. Sousa (1999). Optimum allocation for multiple thrusters. In: Proc. International Society of Offshore and Polar Engineers Conference (ISOPE-99), Brest, France. 